\title{
DIRECT SUMMAND CONJECTURE AND DESCENT FOR FLATNESS
}

\author{
TAKEO OHI \\ (Communicated by Wolmer V. Vasconcelos)
}

\begin{abstract}
We prove the equivalence of the direct summand conjecture and descent of flatness for integral extensions.
\end{abstract}

The purpose of this paper is to prove the following theorem.

Theorem. The following two statements are equivalent:

(D) If $R$ is a regular Noetherian ring and $S(\supset R)$ is a module-finite $R$-algebra, then $R$ is a direct summand of $S$ as an $R$-module.

(F) Let $A$ be a Noetherian ring and let $B$ be an integral extension ring of $A$. Then the extension descends the flatness, that is, if $M$ is an $A$-module such that $M \otimes_{A} B$ is B-flat, then $M$ is A-flat.

The statement (D) is well known as the "Direct summand conjecture" proposed by M. Hochster in [1]. The statement (F) was proposed by M. Raynaud and L. Gruson in [3] (see Questions (1. 4.3) in Part II).

Throughout this paper, all rings are commutative, with identity, all modules are unitary, and ring homomorphisms are assumed to preserve the identity.

Proof. Suppose that (F) holds. Let $R$ be a complete regular local ring, $E$ an injective hull of the residue field of $R$, and $T$ the integral closure of $R$ in an algebraic closure of its fraction field of $R$. Since $E$ is not $R$-flat, $T \otimes_{R} E$ cannot be 0 , which is flat. Since $\operatorname{Hom}_{R}(, E)$ is a faithfully exact functor, we obtain that

$$
\operatorname{Hom}_{R}\left(T \otimes_{R} E, E\right) \simeq \operatorname{Hom}_{R}(T, R) \neq 0 .
$$

Therefore the equivalence of (1) and (4) in Theorem (6.1) of [2] shows that the statement (D) is true.

Conversely, suppose that (D) holds. The statement (F) can be reduced to studying the case where $A$ is a complete regular local ring and $B$ is the integral closure of $A$ in an algebraic closure of its fraction field of $A$ (see p. 67 of [3]). Then Theorem (6.1) in [2] shows that $\operatorname{Hom}_{A}(B, A) \neq 0$. Corollaire 1.2.10 in Part II of [3] together with Remarque 1.2.11 yields the result that $(\mathrm{F})$ is true.

Received by the editors August 18, 1994 and, in revised form, December 21, 1994.

1991 Mathematics Subject Classification. Primary 13B99. 


\section{REFERENCES}

1. M. Hochster, Topics in the homological theory of modules over commutative rings, CBMS Regional Conference Series in Mathematics, number 24, Amer. Math. Soc., Providence, RI, 1975. MR 51:8096

2. Canonical elements in local cohomology modules and the direct summand conjecture, J. Algebra 84 (1983), 503-553. MR 85j:13021

3. M. Raynaud and L. Gruson, Critères de platitude et de projectivité techniques fe 《platification)〉 d'un module, Inventions Math. 13 (1971), 1-89. MR 46:7219

Department of Mathematics, Science University of Tokyo, Shinjuku-ku, Tokyo 162, JAPAN 